\title{
Antidiabetic and Neuroprotective Effects of Trigonella Foenum-graecum Seed Powder in Diabetic Rat Brain
}

\author{
Kumar P. ${ }^{1}$, Kale R. K. ${ }^{1}$, McLean P. ${ }^{2}$, Baquer N. Z. ${ }^{1}$ \\ ${ }^{1}$ School of Life Sciences, Jawaharlal Nehru University, New Delhi, India; \\ ${ }^{2}$ Division of Biosciences, Faculty of Life Sciences, University College London \\ Medical School, London, UK \\ Received May 27, 2011; Accepted January 16, 2012.
}

Key words: Alloxan diabetes - Antioxidant enzymes - Intrasynaptosomal calcium levels - Membrane linked ATPases - Neurolipofuscin - Trigonella foenum-graecum seed powder

\begin{abstract}
Trigonella foenum-graecum seed powder (TSP) has been reported to have hypoglycemic and hyperinsulinemic action. The objective of the study was to examine the antidiabetic and neuroprotective role of TSP in hyperglycemiainduced alterations in blood glucose, insulin levels and activities of membrane linked enzymes $\left(\mathrm{Na}^{+} \mathrm{K}^{+} \mathrm{ATPase}, \mathrm{Ca}^{2+} \mathrm{ATPase}\right.$ ), antioxidant enzymes (superoxide dismutase, glutathione S-transferase), calcium $\left(\mathrm{Ca}^{2+}\right)$ levels, lipid peroxidation, membrane fluidity and neurolipofuscin accumulation in the diabetic rat brain. Female Wistar rats weighing between 180 and $220 \mathrm{~g}$ were made diabetic by a single injection of alloxan monohydrate (15 mg/100 g body weight), diabetic rats were given $2 \mathrm{IU}$ insulin, per day with 5\% TSP in the diet for three weeks. A significant increase in lipid peroxidation was observed in diabetic brain. The increased lipid peroxidation following chronic hyperglycemia was accompanied with a significant increase in the neurolipofuscin deposition and $\mathrm{Ca}^{2+}$ levels with decreased activities of membrane linked ATPases and antioxidant enzymes in diabetic brain. A decrease in synaptosomal membrane fluidity may influence the activity of membrane linked enzymes in diabetes. The present study showed that TSP treatment can reverse the hyperglycemia induced changes to normal levels in diabetic rat brain. TSP administration amended effect of hyperglycemia on alterations in lipid peroxidation, restoring membrane fluidity, activities of membrane bound and antioxidant enzymes, thereby ameliorating the diabetic complications.
\end{abstract}

This study was supported from Council of Scientific and Industrial Research in the form of Junior and Senior Research Fellowship, and by the grant from Indian Council of Medical Research and University Grant Commission.

Mailing Address: Prof. Najma Zaheer Baquer, School of Life Sciences, Jawaharlal Nehru University, New Delhi 110067, India; Phone: +911 126704 501; Fax: +911 126104 865; e-mail: epardeep@gmail.com

(c) Charles University in Prague - Karolinum Press, Prague 2012 


\section{Introduction}

Trigonella foenum-graecum Linn. (Leguminosae) commonly known as fenugreek, is an annual herb, widely grown in India, Egypt, and Middle Eastern countries (Alarcon-Aguilara et al., 1998). It has been extensively used as a source of antidiabetic compound, from its seeds, leaves and extracts in different model systems (Srinivasan, 2006; Losso et al., 2009; Baquer et al., 2011). Preliminary animal and human trials suggest possible hypoglycemic and anti hyperlipidemic properties of oral TSP on type-1 and type-2 DM patients and experimental diabetic animals (Mohammad et al., 2006; Hannan et al., 2007; Yadav et al., 2010). The chemical constituents of TSP include volatile oils, alkaloids, saponins, sapogenins, flavonoids and mucilage. Broca et al. (1999) and Sauvaire et al. (1998) suggested the improvement of the diabetic state, of streptozotocin treated rats, at least partly from a direct stimulating effect of 4-hydroxyisolecuine, an amino acid extracted and purified from fenugreek seeds, which displays in vitro an insulinotropic activity on beta cell function.

The neurological consequences of diabetes mellitus in the central nervous system are now receiving considerable attention (Baquer et al., 2009; Kamboj et al., 2009). Hyperglycemia during diabetes has been shown to generate free radicals, with concomitant increase in intracellular $\mathrm{Ca}^{2+}$ levels with correlated increase in cellular lipid peroxidation of the synaptosomal membrane and inhibition of $\mathrm{Ca}^{2+}$ ATPase activity (Evcimen et al., 1999; Pekiner et al., 2005; Kamboj et al., 2009).

Disturbances in membrane linked ATPases and antioxidant enzymes activities play a role in the pathogenesis of the acute and chronic complications of diabetes (Raza et al., 2004; Tripathi and Chandra, 2009). Lipid peroxidation of the neuronal membrane causes loss of membrane fluidity and inhibition of membrane linked enzymes, increased neurolipofuscin deposition leading to diminished neurotransmitter uptake and loss of membrane asymmetry (Kamboj et al., 2009; Baquer et al., 2011).

Previous studies from our laboratory have demonstrated that oral administration of Trigonella foenum-graecum seed powder to diabetic animals can lower blood glucose levels and partially restore the activities of key enzymes of carbohydrates and lipid metabolism to near normal levels in various animal models of diabetes (Raju et al., 2001; Preet et al., 2005; Mohammad et al., 2006; Baquer et al., 2011; Kumar et al., 2011b, c).

The aim of the present study was to investigate the anti-diabetic potential of TSP on physiological and biochemical parameters like insulin and glucose levels, membrane linked ATPases, antioxidant enzymes, lipid peroxidation, membrane fluidity, intracellular calcium levels and neurolipofuscin accumulation in rat brain.

\section{Material and Methods}

Animals

Adult female albino rats of the Wistar strain, weighing 180-220 g were used for all the experiments. Animals were kept in the animal house maintained at temperatures 
of $22-26{ }^{\circ} \mathrm{C}$ and relative humidity of $55 \%$ with 12 hours dark and light cycle. The animals were fed standard chow (Hindustan Lever Ltd., India) and given tap water ad libitum with the time of treatment. All the animal procedures were approved by the Institutional Animal Ethical Committee (IAEC) of Jawaharlal Nehru University, New Delhi, India.

Induction of diabetes

A group of $60-70$ overnight-starved rats were made diabetic by a single subcutaneous injection of alloxan monohydrate $(15 \mathrm{mg} / 100 \mathrm{~g}$ body weight) freshly prepared in $0.154 \mathrm{M}$ sodium acetate buffer ( $\mathrm{pH} 4.5)$ according to the method of Raju et al. (2001). The alloxan induced diabetic rats were injected i.p. with 2 IU of protamine zinc insulin for the next 7 days; this procedure decreased the mortality of the diabetic animals. The severity of diabetes was checked in alloxan diabetic rats by using glucose strips (Diastix, Bayer Diagnostic, India). Control animals were given only the vehicle.

\section{Experimental design}

Animals were divided into four groups of six rats each. Group I - control (C), Group II - diabetic untreated (D), Group III - diabetic treated with insulin (D+I) and Group IV - diabetic treated with TSP $(D+T)$. Protamine zinc insulin (2 IU) suspension was administered intraperitoneally to diabetic animals $(D+I)$, each day for 21 days. The diabetic treated with TSP $(D+T)$ were given $5 \%$ finely powdered Trigonella seeds in powered rat feed ( $5 \mathrm{~g}$ of dry TSP was mixed with $95 \mathrm{~g}$ of powdered rat feed), each day for 21 days. Seeds of Trigonella foenum-graecum (AGMARK BRAND) were purchased from the local market, New Delhi, India. The plant material was identified in the literature of Ayurveda and by local experts of herbal gardens and further taxonomically validated. The most effective dose of $5 \%$ TSP in the diet has been determined in previous studies (Raju et al., 2001; Mohammad et al., 2006; Baquer et al., 2011; Kumar et al., 2011b, c).

Preparation of homogenate and subcellular fractions Animals were sacrificed by cervical dislocation. Brains of control, diabetic and treated rats were rapidly excised, and washed with chilled normal saline. The tissues homogenates and subcellular fractions were prepared as described earlier, the pellet obtained at 12,000 g containing synaptosomes and mitochondria were used for enzyme assays (Kumar et al., 2011a, b).

Biochemical assays

Membrane ATPases $\mathrm{Na}^{+} / \mathrm{K}^{+}$ATPase and $\mathrm{Ca}^{2+}$ ATPase activities were measured in the synaptosomes according to the method of Mayanil et al. (1982) and Desaiah et al. (1985) respectively. The specific activity of the enzyme is expressed as $\mu \mathrm{mol} \mathrm{Pi}$ released/mg protein/min. 
Antioxidant enzymes The method of Marklund and Marklund (1974) was used to measure the activity of the superoxide dismutase (SOD) with some modification. The specific activity of SOD is expressed as units per $\mathrm{mg}$ protein per minutes. Glutathione S-transferase (GST) was assayed according to the method of Habig et al. (1974). The specific activity of GST is expressed as $\mu \mathrm{mol}$ of 1 -chloro2,4 dinitrobenzene-glutathione (CDNB-GSH) conjugate formed/min/mg protein.

Measurement of lipid peroxidation, membrane fluidity and intrasynaptosomal calcium levels The formation of malondialdehyde (MDA), an end product of fatty acid peroxidation was measured by the method of Genet et al. (2002). Membrane fluidity was measured in the synaptosomes as described previously by Kumar et al. (2011a). Intrasynaptosomal calcium ion concentration was determined by the dual wavelength method described by Grynkiewicz et al. (1985) using calcium sensitive fluorescent probe Fura-2AM. Fluorescence intensity of Fura-2 in crude synaptosomes was measured using Cary Eclipse Spectrofluorimeter (Varian, Palo Alto, USA) with the filters set at $510 \mathrm{~nm}$ for emission and $340 / 380 \mathrm{~nm}$ for excitation.

Measurement of protein, blood glucose and insulin levels Protein was estimated in brain subcellular fractions by the method of Bradford (1976) using bovine serum albumin (BSA) as standard. Blood glucose was estimated by Glucose Enzokit from Ranbaxy Laboratories India, using glucose oxidase method. Serum insulin concentration was measured by enzyme immunoassay using the Mercodia Ultrasensitive Rat Insulin ELISA kit (Mercodia, Uppsala, Sweden).

Histochemical localization and distribution of neurolipofuscin Intraneuronal lipofuscin accumulation has been measured in three brain regions, namely in the cerebral hemispheres, cerebellum and brain stem by fluorescence microscopy as described by Kumar et al. (2011a).

\section{Statistical analysis}

Results were analyzed by means of Prism 5.0 (GraphPad, San Diego, CA, USA). All data were calculated as means \pm SEM of $4-6$ separate values. The ANOVA test followed by Dunnet Multiple Comparison test was employed for statistical comparison between control and various experimental groups. Values with $p<0.05$ were considered as statistically significant.

\section{Chemicals}

All purified enzymes, coenzymes, substrates, standards and buffers were from Sigma Chemicals Company, USA. All other chemicals were of analytical grade and were from SRL and Qualigens, India. 


\section{Results}

Effect of TSP on general parameters

There was a significant decrease in the body weight of the diabetic groups $(p<0.01)$ as compared to controls. After 21 days of insulin and TSP administration, the body weights of diabetic rats increased as compared to control and diabetic groups. The changes in brain weight from control and diabetic groups were not significantly different after 21 days of induction of diabetes. There was a fivefold increase $(p<0.001)$ in blood glucose concentration in alloxan diabetic rats when compared to control rats. Three weeks of treatment with insulin and TSP separately resulted in a significant $(p<0.05)$ reduction in hyperglycemia in the diabetic rats. There was a significant $(p<0.05)$ decrease in insulin levels in the diabetic group when compared with the control. TSP treatment increased the insulin levels in diabetic rats to almost $80 \%$ of the control levels. The protein contents of brain fractions were not significantly affected by diabetes and various antidiabetic treatments. Results are presented in Table 1.

Effect of TSP on membrane linked enzymes $\mathrm{Na}^{+} \mathrm{K}^{+}$ATPase activity was significantly reduced by $28 \%(\mathrm{p}<0.01)$ in synaptosomes of diabetic animals. The treatment of diabetic animals with insulin $(D+I)(p<0.05)$ and TSP $(D+T)(p<0.01)$ separately significantly brought the decreased activity of $\mathrm{Na}^{+} \mathrm{K}^{+}$ATPase to control levels. The induction of diabetes resulted in a decrease by $41 \%(P<0.01)$ in the activity of $\mathrm{Ca}^{2+}$ ATPase in synaptosomes of diabetic animals. The treatment of diabetic animals with insulin and TSP separately brought the decreased activity of $\mathrm{Ca}^{2+}$ ATPase almost to control levels. There was a significant

\section{Table 1 - Changes in physiological parameters, protein, glucose and insulin levels of control (C), diabetic (D), and diabetic rats treated with insulin $(D+I)$ and Trigonella $(D+T)$}

\begin{tabular}{lcccc}
\hline Parameters & $\mathrm{C}$ & $\mathrm{D}$ & $\mathrm{D}+\mathrm{I}$ & $\mathrm{D}+\mathrm{T}$ \\
\hline Body weight $(\mathrm{g})$ & $236 \pm 13$ & $145 \pm 20^{* *}$ & $198 \pm 11$ & $202 \pm 18$ \\
\hline Brain weight $(\mathrm{g})$ & $1.62 \pm 0.30$ & $1.55 \pm 0.25$ & $1.64 \pm 0.28$ & $1.58 \pm 0.29$ \\
\hline Brain weight/100 g & & & & \\
body weight & $0.77 \pm 0.13$ & $1.11 \pm 0.15^{* * *}$ & $0.81 \pm 0.09$ & $0.78 \pm 0.10$ \\
\hline Protein $(\mathrm{mg} / \mathrm{g})$ & & & & \\
Whole homogenate & $81.2 \pm 11.3$ & $86.1 \pm 6.7$ & $91.3 \pm 5.1$ & $88.2 \pm 4.6$ \\
Supernatant & $51.86 \pm 1.87$ & $35.56 \pm 0.95$ & $46.20 \pm 2.42$ & $41.19 \pm 0.78$ \\
Synaptosomes & $18.92 \pm 0.25$ & $17.75 \pm 0.07$ & $19.85 \pm 0.38$ & $18.42 \pm 0.15$ \\
\hline Glucose $(\mathrm{mg} / \mathrm{dl})$ & $87 \pm 6.80$ & $432 \pm 16.21^{*}$ & $103 \pm 8.84$ & $129 \pm 9.71 * * *$ \\
\hline Insulin $(\mathrm{pmol} / \mathrm{l})$ & $750 \pm 14$ & $192 \pm 16^{* * *}$ & $1760 \pm 34^{* *}$ & $630 \pm 25$ \\
\hline
\end{tabular}

Each value is a mean of \pm SEM of five or more separate values from two to three separate experiments. The comparisons of experimental values are with the control values. Statistical significance: ${ }^{*} p<0.001,{ }^{* *} p<0.01, * * * p<0.05$ 
$(\mathrm{p}<0.05)$ increase in $\mathrm{Ca}^{2+}$ ATPase in $(\mathrm{D}+\mathrm{T})$ treated group as compared to control groups. Results are presented in Table 2.

Effect of TSP on antioxidant status

The changes in antioxidant enzymes (SOD and GST) activities in the cytosolic fractions in brain of diabetic and diabetic rats treated with insulin and TSP are shown in Table 2. The induction of diabetes resulted in a significant decrease by $21 \%(p<0.001)$ in the activity of SOD in diabetic animals. The treatment of diabetic animals with insulin $(D+I)$ and TSP $(D+T)$ separately brought the decreased activity of SOD to almost control levels. GST activity was significantly reduced by $45 \%(p<0.01)$ of diabetic animals. The treatment of diabetic animals with insulin and TSP separately brought the decreased activity of GST to almost control levels.

\section{Effect of TSP on lipid peroxidation}

Results in the formation of malondialdehyde (MDA) in control, diabetic and diabetic rats treated with insulin $(D+I)$ and TSP $(D+T)$, are summarized in Table 2. The diabetic rats showed a significant increase of $78 \%(p<0.01)$ in the MDA formation in the brain whole homogenate fractions. Treatment of diabetic rats with insulin, and TSP reversed the increased lipid peroxide formation.

Table 2 - Changes in membrane ATPases, antioxidant enzymes,
membrane fluidity, intrasynaptosomal calcium levels and lipid
peroxidation in brains of control (C), diabetic (D), and diabetic rats
treated with insulin (D+I) and Trigonella $(D+T)$

\begin{tabular}{lcccc}
\hline Parameters & C & D & D+I & D+T \\
\hline Membrane ATPases & $0.534 \pm 0.009$ & $0.388 \pm 0.002^{* *}$ & $0.510 \pm 0.005^{* * *}$ & $0.494 \pm 0.008^{* * *}$ \\
$\mathrm{Na}^{+} \mathrm{K}^{+}$ATPase & $0.62 \pm 0.03$ & $0.39 \pm 0.05^{* *}$ & $0.56 \pm 0.03$ & $0.48 \pm 0.02^{* * *}$ \\
$\mathrm{Ca}^{2+}$ ATPase & & & & \\
\hline Antioxidant enzymes & & & \\
$(\mathrm{U} / \mathrm{mg}$ protein/min) & & & & \\
Superoxide dismutase & $8.93 \pm 0.61$ & $6.37 \pm 0.24^{*}$ & $8.41 \pm 0.29$ & $8.21 \pm 0.35$ \\
Glutathione S-transferase & $3.42 \pm 0.35$ & $1.87 \pm 0.08^{* *}$ & $3.05 \pm 0.19$ & $2.65 \pm 0.28$ \\
\hline Lipid peroxidation & $1.38 \pm 0.28$ & $2.47 \pm 0.18^{*}$ & $1.53 \pm 0.11$ & $1.62 \pm 0.07$ \\
\hline Polarization (P) & $0.191 \pm 0.004$ & $0.247 \pm 0.002^{* *}$ & $0.197 \pm 0.005$ & $0.204 \pm 0.006^{* *}$ \\
\hline Intrasynaptosomal & $181 \pm 26$ & $438 \pm 21^{* *}$ & $220 \pm 18$ & $275 \pm 25^{* * *}$ \\
calcium (nM) & & & & \\
\hline Eal & & & &
\end{tabular}

Each value is a mean of \pm SEM of five or more separate values from two to three separate experiments. The comparisons of experiment values are with the control values. Statistical significance: ${ }^{*} p<0.001,{ }^{* *} p<0.01,{ }^{* * *} p<0.05$. $\mathrm{Na}^{+} \mathrm{K}^{+}$ATPase and $\mathrm{Ca}^{2+}$ ATPase activities are expressed as $\mu \mathrm{mol}$ Pi released $/ \mathrm{mg}$ protein/min. Lipid peroxidation expressed as nmoles of MDA per mg protein 


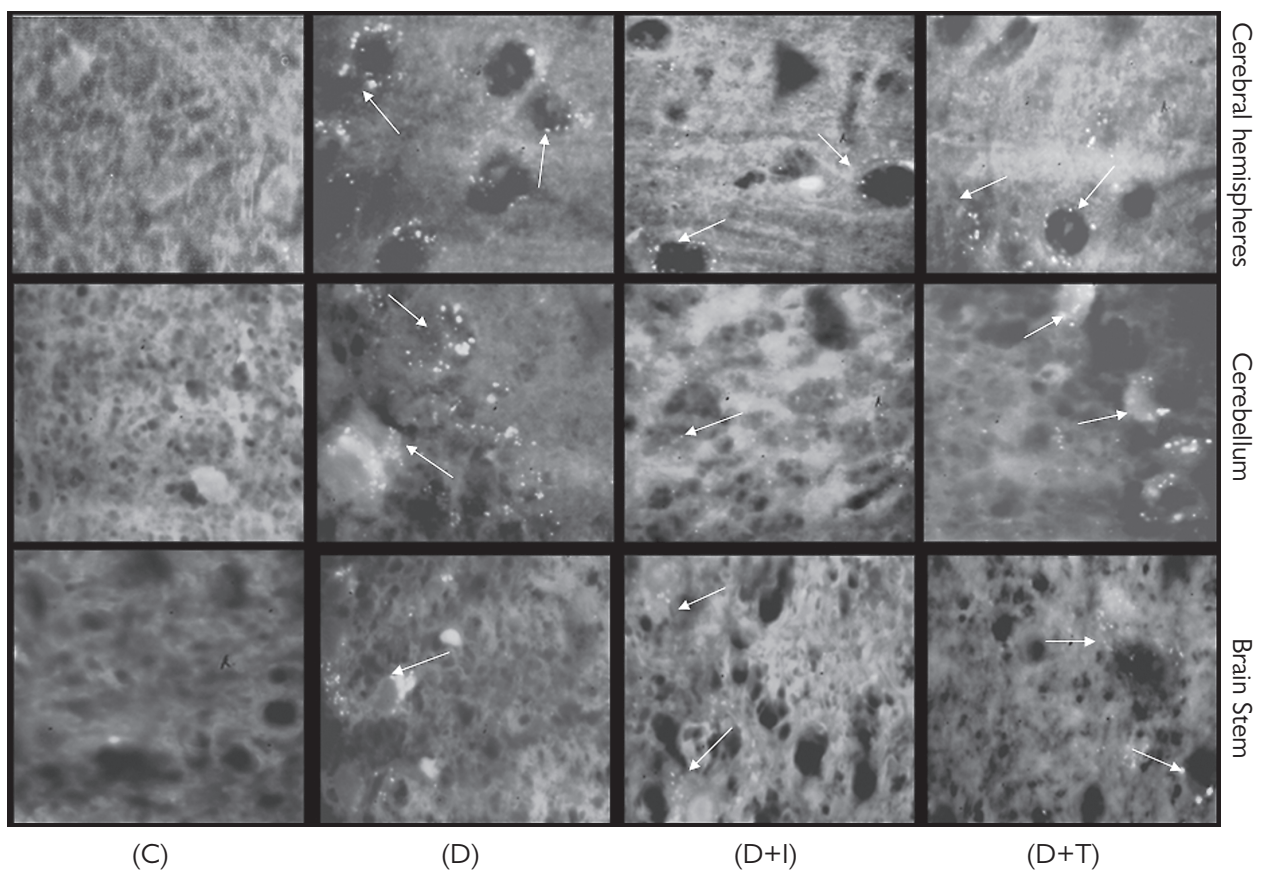

Figure 1 - The neurolipofuscin accumulation (yellow autofluoresence shown by white arrowheads) in cerebral hemispheres, cerebellum and brain stem of control $(C)$, diabetic $(D)$ and diabetic rats treated with insulin $(D+I)$ and Trigonella $(D+T)$.

Effect of TSP on synaptosomal membrane fluidity The DPH fluorescence polarization $(P)$ values were increased in 21 days diabetic groups when compared with the control groups, showing a significant $(p<0.01)$ decrease in the membrane fluidity in the diabetic brain. Treatment of the diabetic animals with insulin $(D+l)$ and TSP $(D+T)(p<0.01)$ restored the changes in the membrane fluidity compared with diabetic and control groups. Results are presented in Table 2.

\section{Effect of TSP on intrasynaptosomal calcium homeostasis}

The changes in intrasynaptosomal calcium level and $\mathrm{Ca}^{2+}$ ATPase activity from brain synaptosomes of diabetic and diabetic rats treated with insulin $(D+I)$ and TSP $(\mathrm{D}+\mathrm{T})$ are shown in Table 2. The intrasynaptosomal calcium levels were found to be significantly increased by 2.5 fold $(p<0.01)$ in the diabetic group. Treatment of the diabetic animals with insulin and TSP restored the changes in the intrasynaptosomal calcium levels when compared with diabetic groups bringing it closer to the controls. There was a significant $(p<0.05)$ decrease in calcium levels in diabetic treated with TSP when compared with the controls. 


\section{Effect of TSP on intraneuronal lipofuscin}

The neurolipofuscin deposition increased with diabetes in all three brain regions i.e. cerebral hemispheres, cerebellum and brain stem as compared to the controls. Treatment of diabetic animals with insulin and TSP decreased lipofuscin deposition in neurons and also showed an increase in the number of neurons without lipofuscin in the all three different brain regions when compared with the controls. The results are presented in the Figure 1.

\section{Discussion}

The experimental animal model of diabetes mellitus of the present study is similar to type-1 diabetes, in which insulin secretion is defective. Hyperglycemia during diabetes has been shown to generate free radicals, increase damage to membranes and decrease antioxidant status (Kumar and Menon, 1993; Preet et al., 2005). Present results showed that three weeks treatment of the diabetic group with insulin, and TSP separately resulted in a marked reduction in hyperglycemia in the diabetic animals (Mohammad et al., 2006; Baquer et al., 2011; Kumar et al., 2011b, c).

$\mathrm{Na}^{+} \mathrm{K}^{+}$ATPase and $\mathrm{Ca}^{2+}$ ATPase play a role in the fine tuning of neuron functions which are decreased in diabetes due to oxidative stress and membrane damages (Pekiner et al., 2005; Kamboj et al., 2009). A decrease in $\mathrm{Ca}^{2+}$ ATPase in diabetic brain could be due to excessive nonenzymatic glycation of the enzyme itself or of calmodulin (Kamboj et al., 2009). TSP and insulin treatment restored the altered $\mathrm{Ca}^{2+}$ ATPase activity to control levels. TSP treatment decreased the oxidative stress and lipid peroxidation. In earlier studies, $\mathrm{Ca}^{2+}$ dependent ATPase activity in liver homogenate of alloxan diabetic rats in the presence of $\mathrm{Fe}^{2+} /$ ascorbate was protected by aqueous extract of Trigonella seed powder (Anuradha and Ravikumar, 2001). A reduction in the production of free radicals and lipid peroxides formation can prevent the decreased membrane ATPases (Kamboj et al., 2009).

A decrease in the antioxidant enzyme activities (SOD and GST) showed that diabetic brain can lead to an excess availability of $\mathrm{O}^{2-}$ and $\mathrm{H}_{2} \mathrm{O}_{2}$ in the biological systems, which in turn generate hydroxyl radicals resulting in initiation and propagation of lipid peroxidation (Kamalakkannan and Stanely, 2006). Administration of TSP increased the activities of SOD and GST in diabetic brain, as TSP includes natural products known to show a range of antidiabetic effects (Yadav et al., 2010) and antioxidant properties (Genet et al., 2002; Tripathi and Chandra, 2009; Baquer et al., 2011).

The formation of MDA was significantly increased in diabetic cerebral cortex, as reported (Kumar and Menon, 1993; Baquer et al., 2011). The decrease in membrane fluidity in synaptosomes from the diabetic brain could be due to the peroxidation of membrane phospholipids through free radicals, generated by persistent hyperglycemia (Anuradha and Ravikumar, 2001; Tripathi and Chandra, 2009). Neurolipofuscin deposition was also increased with experimental diabetes as reported earlier, suggesting that diabetes could have functional change in the neurons 
(Alvarez et al., 2009). Present data suggest that the increase in intrasynaptosomal $\left[\mathrm{Ca}^{2+}\right] \mathrm{i}$ due to oxidative stress may result from the inhibition of the plasma membrane $\mathrm{Ca}^{2+}$ ATPase activity, probably as a result of the alteration of the lipid environment which is essential for the maximal activity (Evcimen et al., 1999). Insulin and TSP treatment to diabetic animals, restored the membrane fluidity, $\mathrm{Ca}^{2+}$ levels and a decrease in lipid peroxides and neurolipofuscin deposition.

The components responsible and the mechanisms by which TSP exerts these effects are not clearly understood. However, as mentioned earlier several studies have shown the presence of steroid saponins in TSP seeds. 4-hydroxyisoleucine, a modified amino acid extracted and purified from Trigonella seeds, increases stimulated insulin secretion. Trigonella is also known to rejuvenate the beta cells of pancreas (Sauvaire et al., 1998). The active component of Trigonella foenum-graecum seeds have been found to be associated with the defatted part of the seeds, rich in fibre containing steroidal saponins and proteins comparable to those of soybean (Al-Habori et al., 2001). Another possible mode of action of TSP was an effect on intestinal carbohydrate digestion and was found to decrease digestion of starch and also glucose absorption both in vivo and in vitro, elucidating a direct inhibitory effect on the digestive enzymes (Hannan et al., 2007; Losso et al., 2009; Baquer et al., 2011; Kumar et al., 2011b, c).

\section{Conclusion}

The data shown in this communication conclude that TSP administration to diabetic rats effectively normalized some of the diabetic aberrations in the brain. Trigonella therefore represents a potentially useful dietary supplement in the treatment of diabetes and a potential source of a new orally active antidiabetic dietary substance.

\section{References}

Alarcon-Aguilara, F. J., Roman-Ramos, R., Perez-Gutierrez, S., Aguilar Contreras, A., Contreras-Weber, C. C., Flores-Saenz, J. L. (1998) Study of the anti-hyperglycemic effect of plants used as antidiabetics.

J. Ethnopharmacol. 61, 101-110.

Al-Habori, M., Raman, A., Lawrence, M. J., Skett, P. (2001) In vitro effect of fenugreek extracts on intestinal sodium-dependent glucose uptake and hepatic glycogen phosphorylase A. Int. J. Exp. Diabetes Res. 2, 91-99.

Alvarez, E. O., Beauquis, J., Revsin, Y., Banzan, A. M., Roig, P., De Nicola, A. F., Saravia, F., (2009) Cognitive dysfunction and hippocampal changes in experimental type 1 diabetes. Behav. Brain Res. 198, 224-230.

Anuradha, C. V., Ravikumar, P. (2001) Restoration on tissue antioxidants by fenugreek seeds (Trigonella Foenum Graecum) in alloxan-diabetic rats. Indian J. Physiol. Pharmacol. 45, 408-420.

Baquer, N. Z., Taha, A., Kumar, P., McLean, P., Cowsik, S. M., Kale, R. K., Singh, R., Sharma, D. (2009) A metabolic and functional overview of brain aging linked to neurological disorders. Biogerontology 10, 377-413.

Baquer, N. Z., Kumar, P., Taha, A., Cowsik, S. M., Kale, R. K., McLean, P. (2011) Metabolic and molecular action of Trigonella foenum-graecum (fenugreek) and trace metals in experimental diabetic tissues. J. Biosci. 36, 383-396. 
Bradford, M. M. (1976) A rapid and sensitive method for the quantitation of microgram quantities of protein utilizing the principle of protein-dye binding. Anal. Biochem. 72, 248-254.

Broca, C., Gross, R., Petit, P., Sauvaire, Y., Manteghetti, M., Tournier, M., Masiello, P., Gomis, R., Ribes, G. (1999) 4-hydroxyisoleucine, experimental evidence of its insulinotropic and antidiabetic properties. Am. J. Physiol. 277, 617-623.

Desaiah, D., Chetty, C. S., Rao, K. S. (1985) Chlordecone inhibition of calmodulin activated calcium ATPase in rat brain synaptosomes. J. Toxicol. Environ. Health 16, 189-195.

Evcimen, N. D., Pekiner, B. D., Nebioglu, S. (1999) Ca(2+)-ATPase activity in streptozotocin-induced diabetic rat kidneys. Diabetes Metab. 25, 399-403.

Genet, S., Kale, R. K., Baquer, N. Z. (2002) Alterations in antioxidant enzymes and oxidative damage in experimental diabetic rat tissues, effect of vanadate and fenugreek (Trigonella foenum-graecum). Mol. Cell. Biochem. 236, 7-12.

Grynkiewicz, G., Poenie, M., Tsien, R. Y. (1985) A new generation of Ca2+ indicators with greatly improved fluorescence properties. J. Biol. Chem. 260, 3440-3450.

Habig, W. H., Pabst, M. J., Jacoby, W. B. (1974) Glutathione-S-transferase: the first enzymatic step in mercapturic acid formation. J. Biol. Chem. 249, 7129-7130.

Hannan, J. M. A., Ali, L., Rokeya, B., Khaleque, J., Akhter, M., Flatt, P., Abdel-Wahab, Y. H. A. (2007) Soluble dietary fibre fraction of Trigonella foenum-graecum (fenugreek) seed improves glucose homeostasis in animal models of type 1 and type 2 diabetes by delaying carbohydrate digestion and absorption, and enhancing insulin action. Br. J. Nutr. 97, 514-521.

Kamalakkannan, N., Stanely, M. P. (2006) Rutin improves the antioxidant status in streptozotocin-induced diabetic rat tissues. Mol. Cell. Biochem. 293, 211-219.

Kamboj, S. S., Chopra, K., Sandhir, R. (2009) Hyperglycemia-induced alterations in synaptosomal membrane fluidity and activity of membrane bound enzymes: beneficial effect of $\mathrm{N}$-acetylcysteine supplementation. Neuroscience 162, 349-358.

Kumar, J. S., Menon, V. P. (1993) Effect of diabetes on levels of lipid peroxides and glycolipids in rat brain. Metabolism 42, 1435-1439.

Kumar, P., Taha, A., Kale, R. K., Cowsik, S. M., Baquer, N. Z. (2011a) Physiological and biochemical effects of $17 \beta$ estradiol in aging female rat brain. Exp. Gerontol. 46, 597-605.

Kumar, P., Kale, R. K., Baquer, N. Z. (2011b) Effects of Trigonella foenum-graecum seed powder on monoamine oxidase, neurolipofuscin, DNA degradation and glucose transporter in alloxan diabetic rat brain. Eur. Rev. Med. Pharmacol. Sci. (in press)

Kumar, P., Kale, R. K., Mukherjee, S., Prakash, K., McLean, P., Baquer, N. Z. (2011c) Antidiabetic effects of Trigonella foenum-graecum seed powder in a rat model. Toxicol. Environ. Chem. 93, 2085-2097.

Losso, J. N., Holliday, D. L., Finley, J. W., Martin, R. J., Rood, J. C., Yu, Y., Greenway, F. L. (2009) Fenugreek bread: a treatment for diabetes mellitus. J. Med. Food 12, 1046-1049.

Marklund, S., Marklund, G. (1974) Involvement of superoxide anion radical in autooxidation of pyrogallol and a convenient assay for superoxide dismutase. Eur. J. Biochem. 47, 469-474.

Mayanil, C. S., Kazmi, S. M., Baquer, N. Z. (1982) Na+,K+-ATPase and Mg2+-ATPase activities in different regions of rat brain during alloxan diabetes. J. Neurochem. 39, 903-908.

Mohammad, S., Taha, A., Akhtar, K., Bamezai, R. N., Baquer, N. Z. (2006) In vivo effect of Trigonella foenum graecum on the expression of pyruvate kinase, phosphoenolpyruvate carboxykinase and distribution of glucose transporter (GLUT4) in alloxan diabetic rats. Can. J. Physiol. Pharmacol. 84, 647-654.

Pekiner, D. B., Evcimen, D. N., Nebioğlu, S. (2005) Diabetes-induced decrease in rat brain microsomal Ca2+-ATPase activity. Cell Biochem. Funct. 23, 239-243.

Preet, A., Gupta, B. L., Siddiqui, M. R., Yadava, P. K., Baquer, N. Z. (2005) Restoration of ultrastructural

Kumar P.; Kale R. K.; McLean P.; Baquer N. Z. 
and biochemical changes in alloxan-induced diabetic rat sciatic nerve on treatment with $\mathrm{Na}_{3} \mathrm{VO}_{4}$ and Trigonella - a promising antidiabetic agent. Mol. Cell. Biochem. 278, 21-31.

Raju, J., Gupta, D., Rao, A. R., Yadava, P. K., Baquer, N. Z. (2001) Trigonella foenum graecum (fenugreek) seed powder improves glucose homeostasis in alloxan diabetic rat tissues by reversing the altered glycolytic, gluconeogenic and lipogenic enzymes. Mol. Cell. Biochem. 224, 45-51.

Raza, H., Ahmed, I., John, A. (2004) Tissue specific expression and immunohistochemical localization of glutathione S-transferase in streptozotocin induced diabetic rats: modulation by Momordica charantia (karela) extract. Life Sci. 74, 1503-1511.

Sauvaire, Y., Petit, P., Broca, C. (1998) 4-hydroxyisoleucine: a novel amino acid potentiator of insulin secretion. Diabetes 47, 206-210.

Srinivasan, K. (2006) Fenugreek (Trigonella foenum-graecum): a review of health beneficial physiological effects. Food Rev. Int. 22, 203-224.

Tripathi, U. N., Chandra, D. (2009) The plant extracts of Momordica charantia and Trigonella foenum-graecum have anti-oxidant and anti-hyperglycemic properties for cardiac tissue during diabetes mellitus. Oxid. Med. Cell. Longev. 2, 290-296.

Yadav, M., Lavania, A., Tomar, R., Prasad, G. B., Jain, S., Yadav, H. (2010) Complementary and comparative study on hypoglycemic and antihyperglycemic activity of various extracts of Eugenia jambolana seed, Momordica charantia fruits, Gymnema sylvestre, and Trigonella foenum graecum seeds in rats. Appl. Biochem. Biotechnol. 160, 2388-2400. 